\title{
Correction to: Electric polarization and depolarization of solder, and their effects on electrical conduction
}

\author{
Wenyi Yang ${ }^{1}$ (D) and D. D. L. Chung ${ }^{1, *}$ (1) \\ ${ }^{1}$ Composite Materials Research Laboratory, Department of Mechanical and Aerospace Engineering, University at Buffalo, The State \\ University of New York, Buffalo, NY 14260-4400, USA
}

Published online:

21 April 2021

(C) Springer Science+Business

Media, LLC, part of Springer

Nature 2021

\section{Correction to: J Mater Sci: Mater Electron (2021) 32:6214-6227 https://doi.org/10.1007/s10854-021-05337-3}

The authors regret a numerical error in calculating the fraction of electrons that participate in the polarization. Thus, Figs. 12 and $15 \mathrm{~b}$ and $\mathrm{c}$ need to be replaced by those shown below. In addition, the following corrections need to be made in the original version of this published article.

In Abstract section line 4, the sentence "The fraction of valence electrons that participate in the initial polarization is of the order of $10^{-9}$." should read as "The fraction of valence electrons that participate in the initial polarization is of the order of $10^{-21}$."

On page 6223, "Results and discussion" section, in para starting with "By using the method describe", the sentence
"The number of valence electrons per atom is 2 for Sn and 1 for Ag." should read as

"The number of valence electrons per atom is 4 for Sn and 1 for Ag.";

The sentence

"The number of valence carriers in the volume is $2.50 \times 10^{23 \prime \prime}$ should read as

"The number of valence carriers in the volume is $4.95 \times 10^{23 \prime \prime}$;

The sentence

"For the initial polarization, the number of electrons per unit volume that participate (up to $1.8 \times 10^{13} \mathrm{~cm}^{-3}$, as shown in Fig. 12) divided by the number of valence electrons per unit volume gives the fraction of valence electrons that participate (up to $2.4 \times 10^{-9}$, as shown in Fig. 12)." should read as

"For the initial polarization, the number of electrons per unit volume that participate (up to $7.8 \times 10^{2} \mathrm{~cm}^{-3}$, as shown in Fig. 12) divided by the

The original article can be found online at https://doi.org/10.1007/s10854-021-05337-3.

Address correspondence to E-mail: ddlchung@buffalo.edu; http://alum.mit.edu/www/ddlchung 


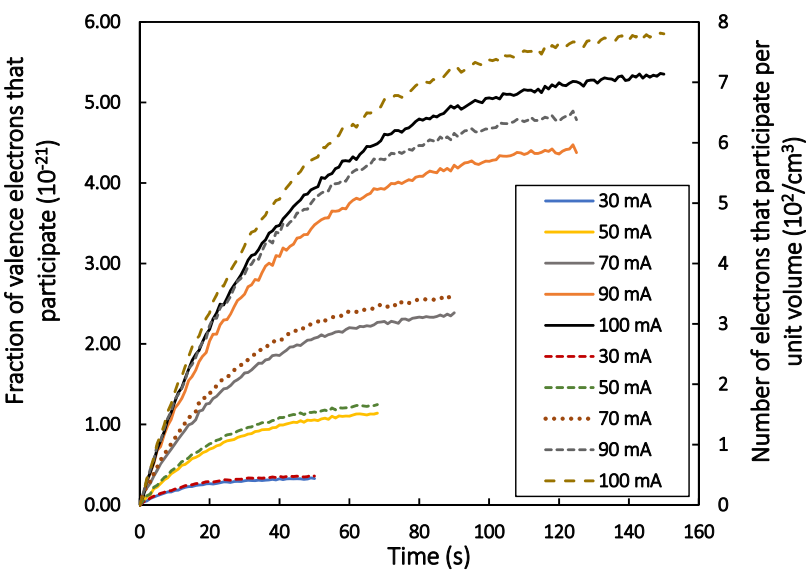

Fig. 12 Fraction of the valence electrons that participate (solidcurves) and number of electrons that participate per unit volume(dashed curves), each vs. time during the initial polarization

number of valence electrons per unit volume gives the fraction of valence electrons that participate (up to $5.4 \times 10^{-21}$, as shown in Fig. 12).";

and the sentence

"This fraction is much higher than the fraction of carriers that participate $\left(10^{-18}\right)$ in case of poly-crystalline graphite [17]." should read as

"This fraction is much lower than the fraction of carriers that participate $\left(10^{-18}\right)$ in case of poly-crystalline graphite [17]."

In the same page, the right column underneath Fig. 13, the sentence

"Nevertheless, the observed abundance of interfaces is believed to contribute to causing the large fraction of carriers that participate in the polarization." should read as

"Nevertheless, the observed abundance of interfaces is believed to contribute to causing the small fraction of carriers that participate in the polarization."

and the sentences

"On the other hand, $n /\left(n_{s}+n_{i}\right)$ (where $n_{s}$ is the number of valence electrons in the solder specimen and $n_{s} \geq n_{i}$ ) increases monotonically with increasing time (Fig. 15c), indicating that the fraction of available electrons (valence electrons + injected electrons) that participate in the polarization increases with increasing time. Since $n_{s} \geq n_{i}, n /\left(n_{s}+n_{i}\right)$ is essentially equal to $n / n_{s} . "$ should read as

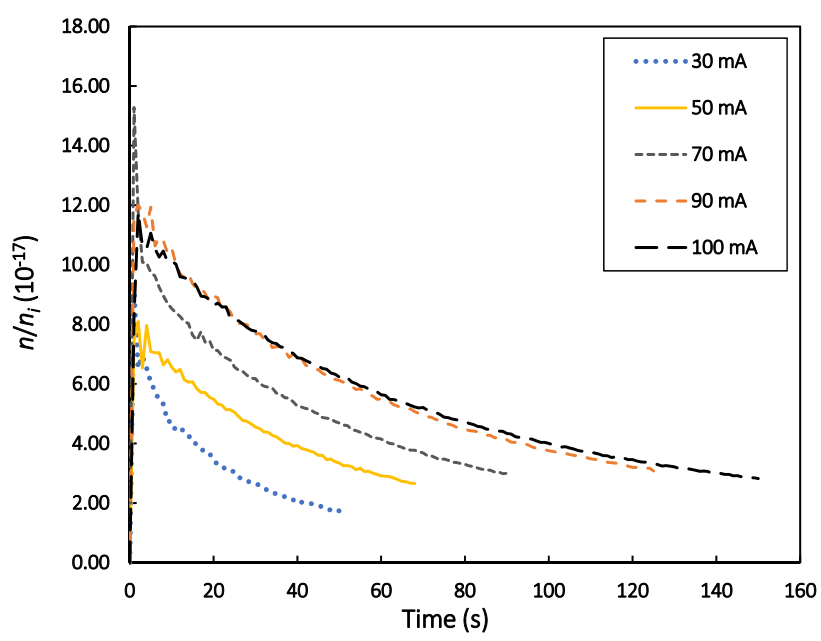

(b)

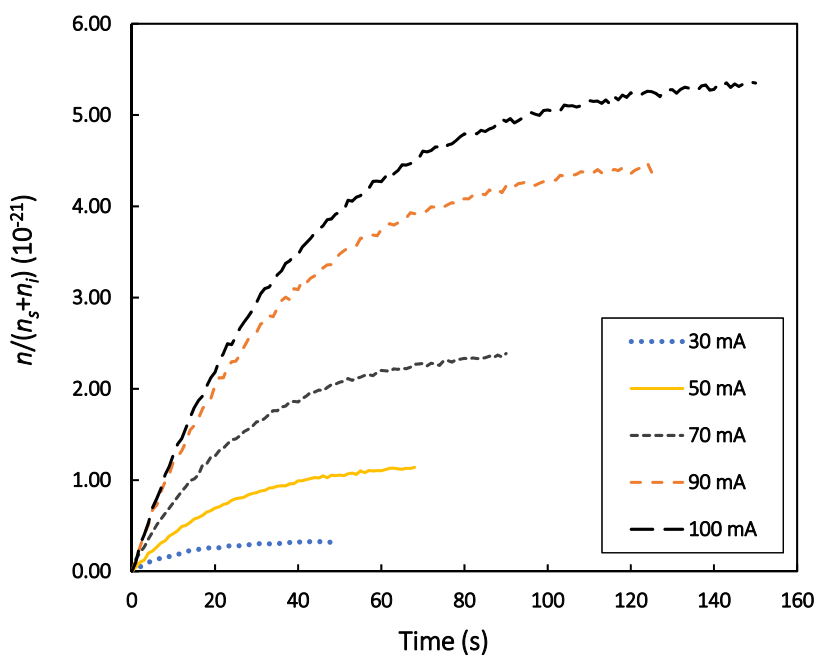

(c)

Fig. $15 \mathbf{b} n / n_{\mathrm{i}}$, where $n$ is thenumber of electrons that participate. c $n /\left(n_{\mathrm{s}}+n_{\mathrm{i}}\right)$, where $n_{\mathrm{s}}$ is thenumber of valence electrons in the solder specimen

"On the other hand, $n /\left(n_{s}+n_{i}\right)$ (where $n_{s}$ is the number of valence electrons in the solder specimen and $n_{s} \gg n_{i}$ ) increases monotonically with increasing time (Fig. 15c), indicating that the fraction of available electrons (valence electrons + injected electrons) that participate in the polarization increases with increasing time. Since $n_{s} \gg n_{i}, n /\left(n_{s}+n_{i}\right)$ is essentially equal to $n / n_{s} . "$

Publisher's Note Springer Nature remains neutral with regard to jurisdictional claims in published maps and institutional affiliations. 